\title{
KLEINIAN GROUPS OF DIVERGENCE TYPE
}

\section{P. J. NICHOLLS}

\begin{abstract}
For a Kleinian group $\Gamma$ acting in the unit ball $B$ we consider the series $\Sigma_{\gamma \in \Gamma}(1-|\gamma(0)|)^{2}$. If the series diverges, $\Gamma$ is said to be of divergence type. From the point of view of the ergodic properties of the group action it is essential to know whether or not $\Gamma$ is of divergence type. If $\Gamma$ is geometrically finite then $\Gamma$ is of divergence type if and only if it is of the first kind. However in the nongeometrically finite case it is not known whether there are any groups of divergence type.

In this paper we give a geometric criterion which is sufficient to ensure divergence type and use this to construct an example of a nongeometrically finite Kleinian group of divergence type.
\end{abstract}

1. Introduction. Let $\Gamma$ be a Kleinian group which acts in the unit ball $B(=\{x$ : $|x|<1\})$ of $R^{3}$. If one considers the ergodic properties of the group action it is critically important to have information regarding the series

$$
\sum_{\gamma \in \Gamma}(1-|\gamma(0)|)^{2}
$$

In his recent important paper on the ergodic properties of discrete group actions Sullivan [7] shows that many natural actions are ergodic if and only if the series (1.1) diverges. We shall say that in this case the group is of divergence type. Given a Kleinian group it is thus a natural question to ask whether or not it is of divergence type. If the group is geometrically finite then it is known that it is of divergence type if and only if it is of the first kind (i.e., every point of $\partial B$ is a limit point for the group)-see, for example, [2]. For nongeometrically finite Kleinian groups much less is known. A rather easy proof shows that any group of the second kind is of convergence type and there are examples to show that the converse is false. However, this author is not aware of a single example in the literature of a nongeometrically finite Kleinian group of divergence type. In view of Sullivan's work it is clearly important to know if there are any such groups. It is the aim of this paper to resolve this question. We shall give a criterion which is sufficient to ensure that a Kleinian group is of divergence type and using this we will exhibit an example of a nongeometrically finite group of divergence type.

In $B$ we use $\rho(x, y)$ to denote the noneuclidean distance which we obtain from the differential

$$
d \rho=2|d x| /\left(1-|x|^{2}\right) .
$$

For a group $\Gamma$ we define $P$, the Dirichlet region centered at the origin, by

$$
P=\{x \in B: \rho(x, 0)<\rho(x, V(0)) \text { all } V \in \Gamma, V \neq I\}
$$

Received by the editors August 5, 1980.

AMS (MOS) subject classifications (1970). Primary 30A58, $20 \mathrm{H} 10$.

Key words and phrases. Kleinian group, geometrically finite, Dirichlet region.

() 1981 American Mathematical Society $0002-9939 / 81 / 0000-0470 / \$ 02.50$ 
where we assume that no element of $\Gamma$ fixes 0 (except the identity). We consider also the isometric spheres. On the assumption that $V \in \Gamma$ does not fix 0 there exist two spheres $I(V)$ and $I\left(V^{-1}\right)$ which have the same euclidean radius, are both orthogonal to $\partial B$ and with the property that the action of $V$ is the composition of inversion in $I(V)$, followed by reflection in the perpendicular bisector of the line segment joining the centers of $I(V)$ and $I\left(V^{-1}\right)$ followed by a rotation about the line through the origin and the center of $I\left(V^{-1}\right)$. It is known that the interior of the set of points of $B$ outside all isometric spheres belonging to transforms in $\Gamma$ is a fundamental polyhedron for $\Gamma$ and it is called the Ford region.

We state now our condition for divergence type. This is the three-dimensional analogue of a result of Laasonen [6, p. 330].

THEOREM 1. Let $\Gamma$ be a Kleinian group with Dirichlet region $P$ centered at the origin. For $0<r<1$ let $\theta(r)$ denote $P \cap\{|x|=r\}$. We write $|\theta(r)|$ for the euclidean area of $\theta(r)$. If $\int_{1 / 2}^{R}(1-r) /|\theta(r)| d r$ is unbounded as $R \rightarrow 1$ then $G$ is of divergence type.

It is important to observe that the condition given in the theorem can only be applied to the Dirichlet region. The reader is referred to [4] for a discussion of how the Laasonen criterion for Fuchsian groups fails if one attempts to apply it to more general fundamental regions. Our next result helps us to recognise the Dirichlet region. Recall that a fundamental region for a Kleinian group $\Gamma$ is said to be locally finite if its $\Gamma$ images do not accumulate in $B$.

THEOREM 2. Let $\Gamma$ be a Kleinian group and let $D$ be a convex, locally finite fundamental polyhedron for $\Gamma$. Then the following are equivalent:

(i) $D$ is the Dirichlet region centered at the origin.

(ii) If $x, V(x)$ belong to $\partial D$, where $V \in \Gamma$, then $|x|=|V(x)|$.

(iii) Each face of $\partial D$ is mapped by an element of $\Gamma$ to another face and these two paired faces belong to spheres of equal radius.

(iv) $D$ is the Ford region.

Using Theorems 1 and 2 we will prove

THEOREM 3. There exists a nongeometrically finite Kleinian group of divergence type.

2. Proof of Theorem 2. The fact that (i) implies (ii) is trivial. To prove (ii) implies (iii) we note that to each face $f$ of $\partial D$ we can associate an element $V \in \Gamma$ and another face $f^{\prime}$ of $\partial D$ with $V(f)=f^{\prime}$. Now if $x \in f$ then $V(x) \in f^{\prime}$ and so we have $|x|=|V(x)|$. It follows from this that $x \in I(V)$ and $V(x) \in I\left(V^{-1}\right)$, so $f$ and $f^{\prime}$ belong to the spheres $I(V), I\left(V^{-1}\right)$ which are of equal radius.

We now show (iii) implies (iv). Consider two paired faces $f, f^{\prime}$ with $f^{\prime}=V(f)$. From the fact that $f, f^{\prime}$ belong to spheres of equal radius it follows immediately that these spheres must be $I(V)$ and $I\left(V^{-1}\right)$. Thus $D$ contains the Ford region and, being a fundamental polyhedron itself, must coincide with the Ford region.

The equivalence of (i) and (iv) is proved in [2, p. 652]. 
3. Proof of Theorem 1. For the Kleinian group $\Gamma$ we denote by $M$ the quotient Riemannian manifold, $M=B / \Gamma$. Recall that the hyperbolic Laplacian on $M$ is

$$
\Delta=\left(1-|x|^{2}\right)^{2} \sum \frac{\partial}{\partial x_{i}}\left(\left(1-|x|^{2}\right)^{-1} \frac{\partial}{\partial x_{i}}\right) \text {. }
$$

A solution of $\Delta u=0$ is said to be hyperbolically harmonic (h.h.). It is known that $B / \Gamma$ has an h.h. Green's function if and only if the series (1.1) converges.

Let $\left\{M_{n}\right\}_{0}^{\infty}$ be an exhaustion of $M$ with connected $M \backslash \bar{M}_{0}$. For each $n$ we let $w_{n}(x)$ be the continuous function on $M$ such that $w_{n}\left|\bar{M}_{0}=0, w_{n}\right| M \backslash M_{n}=1$ and $w_{n}$ is h.h. in $M_{n} \backslash \bar{M}_{0}$. Thus $w_{n}$ is the harmonic measure of $\partial M_{n}$ with respect to $M_{n} \backslash \bar{M}_{0}$. By the maximum principle $w_{n+p} \leqslant w_{n}$, and thus $\lim _{n \rightarrow \infty} w_{n}(x)$ exists on $M$, vanishes on $\bar{M}_{0}$ and is h.h. on $M \backslash \bar{M}_{0}$. This function is denoted by $w(x)$ and is referred to as the harmonic measure of the ideal boundary of $M$ with respect to $M \backslash \bar{M}_{0}$. It is known [5, p. 250] that $M$ has no Green's function if and only if $w \equiv 0$.

Let $\Gamma$ be a Kleinian group of convergence type. We see from our remarks above that the harmonic measure of the ideal boundary is not zero. Now writing $\Gamma=\left\{V_{n}\right\}$ and $x_{n}=V_{n}(0)$ we denote by $S\left(x_{n}, \delta\right)$ an open ball of hyperbolic radius $\delta$ and center $x_{n}$. Then for some $\delta>0$ there exists a function $h$ defined in $B$ with the following properties:

(3.1) $h$ is continuous and bounded in $B$;

(3.2) $h$ is invariant under $\Gamma$;

(3.3) $h$ is h.h. on $B \backslash \cup_{0}^{\infty} \operatorname{cl}\left(S\left(x_{n}, \delta\right)\right)$;

(3.4) if $x \in \operatorname{cl}\left(S\left(x_{n}, \delta\right)\right)$ for some $n$ then $h(x)=0$.

Let $P$ be the Dirichlet region for $\Gamma$ centered at the origin and, for $0<R<1$, let $P(R)=P \cap\{|x|<R\}$ and $\theta(R)=P \cap\{|x|=R\}$. We denote by $P^{*}(R)$ the part of $P(R)$ outside $\operatorname{cl}(S(0, \delta))$. Now apply Green's formula to the function $h$ in the domain $P^{*}(R)$ to obtain ([1] and [5, p. 237])

$$
V(R)=\iiint_{P^{*}(R)} \frac{\operatorname{grad}^{2} h}{1-|x|^{2}} d V=\iint_{\partial P^{*}(R)} h \frac{\partial h}{\partial n} \frac{d A}{1-|x|^{2}}
$$

where $d A$ and $d V$ denote, respectively, the elements of euclidean area and volume at the point $x \in B$.

Note that $h$ is zero on $\partial S(0, \delta)$ and that $h$ has equal and $\partial h / \partial n$ has opposite values at equivalent boundary points. Therefore we have

$$
V(R)=\iint_{\theta(R)} h \frac{\partial h}{\partial n} \frac{d A}{1-R^{2}} .
$$

Using spherical polar coordinates $(r, \theta, \phi)$ we obtain, from (3.5),

$$
V(R)=\int_{\varepsilon}^{R} \iint_{\theta(R)} \frac{\operatorname{grad}^{2} h r^{2} \sin \theta d \theta d \phi d r}{1-r^{2}}
$$

and so

$$
V^{\prime}(R)=\iint_{\theta(R)} \frac{\operatorname{grad}^{2} h}{1-R^{2}} d A
$$


Thus

$$
V^{\prime}(R) \geqslant \iint_{\theta(R)}\left(\frac{\partial h}{\partial r}\right)^{2} \frac{d A}{1-R^{2}} .
$$

Using the Schwartz inequality we obtain, from (3.6),

$$
V(R)^{2}<\left(1-R^{2}\right)^{-1} \iint_{\theta(R)} h^{2} d A \cdot \iint_{\theta(R)}\left(\frac{\partial h}{\partial r}\right)^{2} \frac{d A}{1-R^{2}}
$$

and, from (3.7),

$$
\left(1-R^{2}\right) V(R)^{2}<V^{\prime}(R) \iint_{\theta(R)} h^{2} d A .
$$

Since $h$ is bounded in $B$ we have, for some constant $M$,

$$
\left(1-R^{2}\right) V(R)^{2}<M V^{\prime}(R) \cdot|\theta(R)| .
$$

From (3.8) we see that

$$
\int_{1 / 2}^{R} \frac{\left(1-r^{2}\right)}{|\theta(R)|} d r \leqslant M \int_{1 / 2}^{R} \frac{V^{\prime}(r)}{V(r)^{2}} d r=M\left(\frac{1}{V(1 / 2)}-\frac{1}{V(R)}\right)
$$

and the right-hand side is bounded by $M / V(1 / 2)$ as $R \rightarrow 1$. Thus if $\Gamma$ is of convergence type, the integral $\int_{1 / 2}^{R}(1-r) /|\theta(r)| d r$ remains bounded as $R \rightarrow 1$ and we have proved Theorem 1.

4. Proof of Theorem 3. Let $x=(2 \sqrt{2}-1)^{-1 / 2}$ and $R=2(2+3 \sqrt{2})^{-1 / 2}$. We consider eight spheres $S_{i}, 1 \leqslant i \leqslant 8$, each of radius $R$ and with centers, respectively, $(x,-x, x),(x, x, x),(x,-x,-x),(x, x,-x),(-x,-x, x),(-x, x, x)$, $(-x,-x,-x)$ and $(-x, x,-x)$. Now let $y=(2 \sqrt{2}-1)^{1 / 2}$, and $\rho=(2 \sqrt{2}-2)^{1 / 2}$. We consider six spheres $C_{j}, 1 \leqslant j \leqslant 6$, each of radius $\rho$ and with centers, respectively, $(0,0, y),(0,0,-y),(y, 0,0),(-y, 0,0),(0,-y, 0),(0, y, 0)$. Since $3 x^{2}=1+$ $R^{2}$ and $y^{2}=1+\rho^{2}$ it follows that each of the fourteen spheres meets the unit sphere orthogonally. One can check easily that $C_{i} \cap C_{j}=\varnothing$ if $i \neq j$ and that each sphere $C_{i}$ meets four of the spheres $S_{j}$ each at an angle of $\pi / 2$. Thus $C_{1}$ meets $S_{1}$, $S_{2}, S_{5}, S_{6}$ each at an angle of $\pi / 2$ since $R^{2}+\rho^{2}=2 x^{2}+(x-y)^{2}$. Similarly it can be checked that each sphere $S_{i}$ meets three other spheres $S_{j}$ each at an angle of $\pi / 4$. Thus, for example, $S_{1}$ meets $S_{2}, S_{3}, S_{5}$ each at an angle of $\pi / 4$ since $4 x^{2}=R^{2}(2+\sqrt{2})$.

Let $P$ be the region in $B$ which is exterior to all the spheres $S_{i}, 1 \leqslant i<8$, and $C_{j}$, $1 \leqslant j \leqslant 6$. Then we have

LeMMA 1. With $P$ defined as above, $\bar{P} \subset B$.

Proof. Suppose there is a point of $\partial B$ which is in $\bar{P}$. Let such a point be $(\alpha, \beta, \gamma)$, which we may suppose to be in the first octant. From the fact that $(\alpha, \beta, \gamma)$ is not interior to the spheres $C_{1}, C_{3}, C_{6}$ and $S_{2}$ we deduce that

$$
\begin{gathered}
\alpha y \leqslant 1, \quad \beta y \leqslant 1, \quad \gamma y<1, \\
\alpha+\beta+\gamma<y .
\end{gathered}
$$


Clearly we also have

$$
\alpha \geqslant 0, \quad \beta \geqslant 0, \quad \gamma \geqslant 0 .
$$

Routine calculations show that the function $\alpha^{2}+\beta^{2}+\gamma^{2}$, subject to the constraints (4.1)-(4.3), attains its maximum at the point $(1 / y, y-1 / y, 0)$ and the maximum value is

$$
y^{2}-2+2 / y^{2}=(13-8 \sqrt{2}) /(2 \sqrt{2}-1)<1
$$

so $(\alpha, \beta, \gamma) \notin \partial B$, which is a contradiction. Thus $\bar{P} \subset B$ as required.

We now form seven Moebius transforms which pair the sides of $P$. Let $E_{i}$, $1 \leqslant i<4$, be elliptic transforms each of order eight which map, respectively, $S_{1}$ to $S_{2}, S_{3}$ to $S_{4}, S_{5}$ to $S_{6}$ and $S_{7}$ to $S_{8}$. let $A, B, C$ be three hyperbolic transforms which map, respectively, $C_{1}$ to $C_{2}, C_{3}$ to $C_{4}$ and $C_{5}$ to $C_{6}$.

It is easy to see that the polyhedron $P$ and the set of side pairers defined above satisfy the hypotheses of Poincaré's theorem (see [3, p. 228]). Thus the transforms $E_{i}, 1 \leqslant i \leqslant 4, A, B, C$ generate a Kleinian group $G$ and $P$ is a fundamental polyhedron for $G$. Furthermore, consideration of the edges of $P$ shows that the following are a complete set of relations for $G$ :

$$
\begin{gathered}
E_{1}^{-1} A^{-1} E_{2} A=E_{3}^{-1} A^{-1} E_{4} A=E_{1} B^{-1} E_{3}^{-1} B=E_{2} B^{-1} E_{4}^{-1} B=I, \\
\left(C^{-1} E_{i}\right)^{2}=I, \quad 1<i<4, \\
\left(E_{3} E_{1}^{-1}\right)^{4}=\left(E_{3}^{-1} E_{2}\right)^{4}=\left(E_{2}^{-1} E_{1}\right)^{4}=\left(E_{4}^{-1} E_{3}\right)^{4}=I, \\
E_{i}^{8}=I, \quad 1<i<4 .
\end{gathered}
$$

From our construction and Theorem 2 we see that $P$ is the Dirichlet region for $G$ centered at the origin. From Lemma 1 it follows that the hyperbolic volume of $P$ $(\mathrm{Vol} P)$ is finite.

The group we are interested in is a subgroup of $G$ and is defined as follows. If $V \in G$ we may write $V$ as a word in the generators $E_{i}, 1<i<4, A, B, C$. Define $\exp _{A} V$ to be the sum of the powers of $A$ which appear when $V$ is written as such a word. Set $\Gamma=\left\{V \in G\right.$ : $\left.\exp _{A} V=0\right\}$. Note that $\Gamma$ is well defined, since in each relation for $G$ the powers of $A$ add up to zero. It is clear that the region $P^{*}=\cup_{n=-\infty}^{\infty} A^{n}(P)$ is a fundamental polyhedron for $\Gamma$.

LEMMA 2. The polyhedron $P^{*}$ is the Dirichlet region for $\Gamma$ centered at the origin.

Proof. For each integer $n$ the transform $A^{n}$ is hyperbolic and its axis is the $z$ axis. We observe from our construction that if $S \in\left\{E_{i}, 1<i<4, B, C\right\}$ then $I(S)$ and $I\left(S^{-1}\right)$ are symmetrically located with respect to the $z$ axis $\left(I\left(S^{-1}\right)\right.$ may be obtained from $I(S)$ by a rotation about the $z$ axis). It follows trivially from this observation that if $y \in I(S)$ then

$$
\left|A^{n}(y)\right|=\left|A^{n} S(y)\right| \text {. }
$$

Now consider two equivalent points on $\partial P^{*}$. Clearly these points must be of the form $x$ and $A^{n} S A^{-n}(x)$ for some integer $n$ and $S$ as above. Write $y=A^{-n}(x)$ and observe that $y, S(y)$ are equivalent points on $\partial P$. Since $P$ was the Ford region for $G$ 
we must have $y \in I(S)$. We apply (4.8) and note that $|x|=\left|A^{n} S A^{-n}(x)\right|$. Thus equivalent points on $\partial P^{*}$ are the same distance from the origin and, by Theorem 2 , the proof is complete.

Now $A$ is hyperbolic with multiplier $K$, say, where $K>1$. If $r_{n}$ is the radius of $I\left(A^{n}\right)$ then there exists a constant $M$ so that

$$
r_{n}=K^{n / 2} M /\left(K^{n}-1\right)
$$

by a routine calculation.

If $n$ is so large that $\left(1+r_{n}^{2}\right)^{1 / 2}-r_{n}>R$ then $I\left(A^{n}\right)$ is contained in $\{x$ : $|x|>R\}$, and it follows that $A^{n}(P) \cap\{|x|<R\}=\varnothing$. Thus the hyperbolic volume of $P^{*} \cap\{|x|<R\}$ does not exceed the volume of $P$ times the number of integers $n$ satisfying $\left(1+r_{n}^{2}\right)^{1 / 2}-r_{n}<R$. An easy calculation yields

$$
\operatorname{Vol}\left(P^{*} \cap\{|x|<r\}\right)=O\left(\log \left((1-r)^{-1}\right)\right) \text { as } r \rightarrow 1 \text {. }
$$

With $\theta(r)$ defined by $\theta(r)=P^{*} \cap\{|x|=r\}$ we see that

$$
\operatorname{Vol}\left(P^{*} \cap\{|x|<r\}\right)=\int_{0}^{r} \frac{8|\theta(t)|}{\left(1-t^{2}\right)^{3}} d t .
$$

Since $P^{*}$ is convex, $|\theta(t)| t^{-2}$ is decreasing and so

$$
\operatorname{Vol}\left(P^{*} \cap\{|x|<r\}\right) \geqslant \frac{|\theta(r)|}{r^{2}} \int_{0}^{r} \frac{t^{2} d t}{\left(1-t^{2}\right)^{3}}
$$

From this and (4.9) we see that

and so

$$
|\theta(r)| /(1-r)^{2}(-\log (1-r))=O(1) \quad \text { as } r \rightarrow 1
$$

$$
\int_{1 / 2}^{R} \frac{1-r}{|\theta(r)|} d r \rightarrow \infty \quad \text { as } R \rightarrow 1 .
$$

By Theorem 1, $\Gamma$ is of divergence type as required.

\section{REFERENCES}

1. L. V. Ahlfors, Fundamental polyhedrons and limit point sets of Kleinian groups, Proc. Nat. Acad. Sci. U.S.A. 55 (1966), 251-254.

2. A. F. Beardon and P. J. Nicholls, On classical series associated with Kleinian groups, J. London Math. Soc. 5 (1972), 645-655.

3. B. Maskit, On Poincaré's theorem for fundamental polygons, Adv. in Math. 7 (1971), 219-230.

4. P. J. Nicholls, Fundamental regions and the type problem for a Riemann surface, Math. Z. 174 (1980), 187-196.

5. B. Rodin and L. Sario, Principal functions, Van Nostrand, Princeton, N.J., 1968.

6. L. Sario and M. Nakai, Classification theory of Riemann surfaces, Springer-Verlag, New York, 1970.

7. D. Sullivan, On the ergodic theory at infinity of an arbitrary discrete group of hyperbolic motions (Proc. Stony Brook Conf. Riemann Surfaces and Kleinian Groups, June, 1978), Ann. of Math. Studies, Vol. 97, Princeton Univ. Press, Princeton, N.J., 1981.

Department of Mathematical Sciences, Northern Illinois University, DeKalb, Illinois 60115 